\title{
Subjetividad y normativa en Canguilhem y Foucault
}

\author{
Pierre Macherey* \\ Traducción del francés al español \\ de Luis Alfonso Palau Castaño**
}

Cuando Canguilhem tuvo conocimiento de la primera gran obra de Foucault, Historia de la locura, sobre la que tuvo que escribir un informe en tanto que jurado de tesis, inmediatamente subrayó su carácter innovador, y su importancia, mucho más allá de los límites concedidos a un trabajo especializado que concernía la historia de la psiquiatría; algunos años más tarde, hacía aparecer en la colección Galeno que dirigía en PUF, Nacimiento de la clínica, la obra de Foucault que, sin duda, más le interesó, porque su tema lo concernía de más cerca, y a la que a menudo se refirió en sus propios trabajos ${ }^{1}$. En fin, cuando Les Mots et les choses fue puesto en circulación, le consagró con el título "¿Muerte del hombre o agotamiento del Cogito?", un importante estudio aparecido en 1967 en Critique en el que, tomando su defensa contra sus contradictores o sus censuradores -se estaba entonces en plena querella del humanismo- él elogiaba la "lucidez" del proceder de Foucault, a propósito de la que llegaba hasta sugerir en conclusión que ella podría jugar con respecto a las ciencias humanas un rol comparable al que había jugado la Crítica de la razón pura para las ciencias de la naturaleza. Por lo demás, uno de los últimos escritos de los que Foucault autorizó su publicación fue la retoma de una presentación general del camino de Canguilhem, que había sido redactado en 1978 en el momento en que lo tradujeron en los EE. UU.; ese texto, titulado en su versión definitiva "la

* Pierre Macherey. Subjetividad y normatividad en Canguilhem y Foucault. Ponencia presentada el 1..$^{\circ}$ de junio de 2016 en el marco de una jornada de estudios sobre "Michel Foucault y la subjetivación» (Universidad ParisEst Créteil). Traducción para la revista Ciencias Sociales y Educación realizada en Medellín el 6 de junio de 2016.

** Profesor titular de Historia de la Biología de la Universidad Nacional de Colombia, sede Medellín. Profesor emérito y jubilado de la Facultad de Ciencias Humanas y Económicas. Doctor en Historia y Filosofía de las Ciencias de la Universidad de París I (Sorbona-Panteón). Correo electrónico: lapalau@une.net.co

1 Al final de la parte complementaria, redactada "veinte años después", con la que termina Le normal et le pathologique, Canguilhem señala que "en páginas admirables, conmovedoras, del Naissance de la clinique, Michel Foucault mostró cómo Bichat hizo "girar la mirada médica sobre sí misma, para pedirle a la muerte cuentas de la vida" (Le normal et le pathologique, Paris, PUF/Quadrige, 1988, p. 215). Esta conversión de la mirada que él llama también "eversión", es la que el propio Canguilhem ha tratado de practicar. Los dos libros de Foucault, Histoire de la folie (1961) y Naissance de la clinique (1963) son añadidos como referencia en el Suplemento a la bibliografía de la nueva edición, en 1966, de La connaissance de la vie, lo que subraya la importancia que Canguilhem les concedía. 
Vida: la experiencia y la ciencia", es sin duda uno de los más importantes y de los más pertinentes comentarios que hayan sido consagrados al pensamiento de aquel que, en la conversación, Foucault llamaba en ese momento -sin ironía, y siendo él avaro en este tipo de efusiones- "nuestro viejo maestro"2. Se puede pues decir que Canguilhem y Foucault se han reconocido (en el sentido fuerte del término), e incluso en parte reconocido el uno en el otro a través de intereses y valores que compartían en común; entre ellos se tejió una relación intelectual fuerte que podemos suponer jugó un rol nada despreciable en el desarrollo de sus respectivos pensamientos.

Sin embargo, este reconocimiento estaba lejos de ser evidente; incluso, a primera vista no hubiera debido presentarse, tanto por la diferencia en las posiciones de los protagonistas de esta relación en el campo filosófico, como por la distancia que existía entre algunos de los presupuestos intelectuales que comportaban sus respectivas gestiones ${ }^{3}$. En efecto, Canguilhem pertenecía al linaje de Alain, un filósofo del juicio, del deber-ser y del sujeto que asume -como él lo decía- sus "exigencias", que lo hacen "normativo", tanto como lo pueda; mientras que Foucault, al menos al comienzo, se caracterizaba por su reserva con respecto a una posición subjetivizadora, tendencialmente humanista, orientada por consideraciones privilegiadas de las figuras de la conciencia, cuya consecuencia era que una cuestión como la del deber-ser, en el sentido de un imperativo asumido de manera reflexiva, no tenía a priori sentido para él, por no decir incluso que no tenía ninguno. Por otra parte, Canguilhem era una figura respetada en la Universidad, que si no era propiamente el guardián del templo se había hecho conocer en tanto que profesor, inspector general, miembro o presidente del jurado de la agregación, por el rigor de sus posiciones, que había podido hacer interpretarlas en el sentido de un cierto conformismo ${ }^{4}$;

$2 \quad$ M. Foucault. "La vida: la experiencia y la ciencia". Revista de Metafísica y Moral. 90 año/\#1. Enero-marzo/1985. tr. Paláu, publicada in Sociología 18, Medellín: Universidad Autónoma Latinoamericana, Julio/1995 ..

3 En el libro que le consagró, F. Dagognet señala que "Los amigos de Georges Canguilhem a veces se interrogan -una pregunta que linda con la reprobación- por la estima, incluso según algunos la indulgencia, que tuvo para con los trabajos de Michel Foucault" (Georges Canguilhem filósofo de la vida, in traducciones historia de la biología $n^{\circ}$ 25. tr. Paláu, Medellín: Universidad Nacional de Colombia, nov. de 2003, p. 9). Él prosigue explicando que esa sorpresa que quizá él mismo compartió en un grado o en otro, encuentra su justificación en una interpretación unilateral del pensamiento de Canguilhem; el hilo conductor de su libro es que ese pensamiento presenta "vertientes" contrastadas, la una tendencialmente conservadora, que lo alejaba de Foucault, la otra tendencialmente contestataria que lo acercaba a él. Dagognet subraya a continuación: “ Hostil a la estandarización y a la uniformización de los cuerpos, la tesis sobre Lo normal y lo patológico anticipa los futuros análisis de Michel Foucault; Georges Canguilhem los había anticipado hasta tal punto que estará inevitablemente atraído por ellos, incluso si posteriormente los integrará a otros desarrollos " (ibíd., p. 29) Se puede estar de acuerdo con Dagognet cuando él avanza que la relación entre Canguilhem y Foucault, relación esencialmente compleja, asocia proximidad y alejamiento, en condiciones tales que su tensión no puede ser directamente resuelta. Incluso vayamos más lejos: es esta tensión la que hace fecunda esa relación en la práctica.

4 La mayor parte de las reflexiones y de las intervenciones de Canguilhem a propósito de la enseñanza de la filosofía en los Liceos -enseñanza a la que estaba visceralmente adherido gracias a su recorrido personal-están 
mientras que las relaciones de Foucault con las instituciones estuvieron desde el comienzo marcadas por la desconfianza, lo que las hizo difíciles, por no decir que en ocasiones fueron tumultuosas. Para resumir en una sola palabra esta situación, Canguilhem -lector asiduo de Renouvier, de Lachelier \& de Hamelin, filósofos neo-kantiano reputados entonces como de retaguardia, en los que uno difícilmente imagina que Foucault se haya podido interesar-estaba de lleno en "el sistema", incluso si detentaba dentro de él una posición singular; mientras que Foucault (que había tenido dificultades para digerir su fracaso la primera vez que se presentó al concurso de la agregación en filosofía) adoptaba con respecto a ese "sistema" la actitud de un contestatario, que a lo sumo consiente en ocupar ciertos márgenes, lo que marcó su carrera universitaria (si es que esta expresión es apropiada en su caso) y la hizo seguir una trayectoria bastante imprevisible e irregular, pero no por ello menos brillante.

En estas condiciones, ¿cómo Canguilhem y Foucault lograron entenderse, permaneciendo cada uno firme en sus posiciones respectivas, cuando cada uno por su lado la asumía con el más grande rigor? El presente estudio tiene por hilo conductor la hipótesis siguiente: es en torno a la relación entre lo normativo y lo subjetivo donde se anudó la discusión entre Canguilhem y Foucault. A la pregunta "¿qué es ser sujeto bajo normas?" ellos respondieron, el primero, Canguilhem, razonando del sujeto hacia las normas, por tanto planteando que las verdaderas normas son aquellas que los sujetos ponen en funcionamiento dinámicamente en la medida en que "toman partido"5; el otro, Foucault, razonando de

marcadas por ese conformismo. Canguilhem era sensible a las tentativas de innovación pedagógica pero, en el fondo, él le atribuía a la enseñanza de la filosofía, y al modelo propiamente francés de tal enseñanza -la institución de la "classe de philosophie"- que le confiere a esta una función social eminente, un valor primordial que no estaba dispuesto a cuestionar. Foucault tenía sobre el modelo francés una mirada claramente más distanciada, como lo testifican sus anotaciones al respecto en una entrevista aparecida en 1970 en Le Nouvel Observateur titulada "La trampa de Vincennes", en donde el "juego" del sistema de enseñanza francés es resumido así: "a los alumnos de primaria, la sociedad les da el "leer-y-escribir" (la instrucción); a los del técnico, les ofrece saberes a la vez particulares y útiles; a los de secundaria, que normalmente deben entrar a la facultad, les entrega saberes generales (la literatura, la ciencia), pero al mismo tiempo la forma general de pensamiento que permite juzgar todo saber, toda técnica, y la raíz misma de la instrucción. Les concede el derecho y el deber de "reflexionar"; de ejercer su libertad pero sólo en el orden del pensamiento, de ejercer su juicio, pero sólo en el orden del libre examen" (Dits et Ecrits, t. II, Paris, Gallimard, 1994, p. 69 iver el texto completo Infra como anexo 1, Paláuı). Se subrayaba así el carácter paradójico del régimen de la Sociedad-Escuela tal como se practica en Francia desde el siglo XIX, bajo formas que asocian integración y separación, incorporación y exclusión. Sin condenarla, Canguilhem por su lado estando abierto al espíritu de utopía, consideraba con una cierta perplejidad la experiencia que se llevaba a cabo en el departamento de filosofía de Vincenne.

5 En uno de sus primerísimos escritos, Canguilhem se reclama de una filosofía "de la toma de partido" (" Fascisme et révolution ", Libres Propos, marzo de 1933, Oeuvres complètes, Paris, Vrin 2013, p. 453). Encuentra ahí la justificación filosófica de su compromiso en la Resistencia, siguiendo a Cavaillès, compromiso que responde él mismo a la exigencia de ser "normativo", y no sometido a normas convenidas, cuya fórmula "Trabajo, familia, patria" constituye su expresión caricaturesca. En la teoría como en la práctica, Canguilhem le concede un valor primordial al espíritu de "vigilancia", en el sentido de lo que Alain había llamado las "Vigilias del espíritu"; paradójicamente es la puesta en funcionamiento riguroso de este espíritu de vigilancia el que, en el momento de la guerra, lo alejó de Alain y del grupo de los alainistas (que terminaron acercándose a Vichy). 
las normas al sujeto, por ende, planteando que solo existen sujetos sujetados a normas que tienen sus fuentes en otra parte distinta a una conciencia, instancia de juicio (Foucault). Desarrollando hasta sus últimas consecuencias estas dos opciones de sentido aparentemente inverso, Foucault y Canguilhem no dejaron de acercarse y entenderse, en un espíritu de verdadera connivencia, sin que, sin embargo, sus respectivas posiciones llegaran exactamente a confundirse.

Para darle un contenido preciso a la alternativa que se acaba de esbozar, veamos cómo intervino en el marco de la discusión suscitada por el concepto de episteme. En “¿Muerte del hombre o agotamiento del cogito?”, Canguilhem señala:

No existe en la actualidad filosofía menos normativa que la de Foucault, más ajena a la distinción de lo normal y de lo patológico ${ }^{6}$.

Esta anotación toma toda su importancia cuando uno se recuerda que, en la situación opuesta, Canguilhem hace él mismo pasar al primer plano de su trabajo la consideración de lo normativo, y hace de la separación de lo normal y de lo patológico el objetivo principal de su reflexión. Por consiguiente, uno no se sorprende que plantee la siguiente cuestión en la continuación de su artículo, que a primera vista es una objeción hecha a Foucault:

Tratándose de un saber teórico ¿será posible pensarlo en la especificidad de su concepto sin referencia a ninguna norma? ${ }^{7}$

Pensar un saber teórico en la especificidad de su concepto, reintegrándolo para ello a la dinámica propia del conocimiento científico que es una actividad completa cuyo desenvolvimiento es histórico, no es, en efecto, aprehenderlo en un plano de generalidad, como orden teórico puro, indiferenciado y neutro, cuyo desarrollo unívoco estaría sometido a una ley objetiva de la cual él saca su carácter de necesidad, sino que es seguir el camino efectivo en el curso del cual se elaboraron poco a poco, de manera imprevisible e irregular, los conceptos que permiten tratar problemas específicos, como, por ejemplo, el del comportamiento reflejo (tema de la tesis que Canguilhem preparó bajo la dirección de Bachelard ${ }^{8}$ ); ahora bien, hacer la historia de un concepto es establecer el

Art. cit., Revista ECO.

Ibíd.

8 La idea directriz del estudio que Canguilhem consagró a La formación del concepto de reflejo en los siglos XVII y XVIII (Paris, PUF, 1955) es que, contrariamente a lo que uno se figura generalmente porque se considera a priori que las cosas hubieran debido pasar así, el concepto de reflejo no apareció en el contexto de un estudio del funcionamiento de los organismos apoyado en un presupuesto mecanicista (Descartes), sino por el contrario en un contexto vitalista (Willis). Se encuentra acá una aplicación del principio general según el cual la historia de las ciencias, que es una historia en el pleno sentido del término, no puede ser forzada a existir en el plano de una reducción racional que no le asignara ningún sitio a acontecimientos de pensamiento; ella no es una historia de las teorías y de su encadenamiento, historia en la que no hay lugar para tales acontecimientos, sino una historia de los conceptos cuya formación está sometida a los azares, lo que la hace por una parte imprevisible. 
inventario de las elecciones que han sido hechas en tal o cual momento por tal o cual científico que, descartando para ello otras, las ha privilegiado, de alguna manera las ensaya; y no se ve cómo esas escogencias hubieran podido ser efectuadas en ausencia de normas, por tanto de principios de evaluación, asumidos con toda responsabilidad por los que de ellos se reclaman, que habrían fijado las orientaciones en respuesta a esperas y a exigencias de orden axiológico, y no únicamente lógico. De hecho, la historia de las ciencias practicada por Canguilhem, que es, a la vez, una historia de los problemas y una historia de los conceptos que permiten formular esos problemas a la espera de su solución que no estaría de ninguna manera prefigurada desde el comienzo en su enunciado, es, no un desenvolvimiento predeterminado en y por su estructura de partida, sino una sucesión de experimentos y de aventuras teóricas llevadas a cabo por los científicos, auténticos sujetos de saber que, a medida que -se van volviendo bajo sus propios riesgos y peligros, normativos- han decidido dirigirse en tal o cual sentido y sacar todas las consecuencias de dicha elección. Es pues pesando cuidadosamente sus palabras que Canguilhem tituló la conferencia que pronunció en 1964, en conmemoración del cuarto centenario del nacimiento de Galileo: "la Significación de la obra y la Lección del hombre"9. Una cosa es la significación de la obra, es decir, el valor de verdad o el grado de cientificidad, que le pueden ser atribuidas a las tesis que ella vehicula; y otra es la lección del hombre, es decir, el compromiso de este con un trabajo de pensamiento jalonado a punta de tomas de partido, como el rechazo del geocentrismo, una orientación bien arriesgada cuya responsabilidad fue asumida personalmente por Galileo. Canguilhem muestra en su conferencia de 1964 que, comprometiéndose por esta vía -una elección que sabemos le costó personalmente muy caro-Galileo "estaba en la verdad" (se lo puede afirmar hoy) sin que esto signifique propiamente hablando que él decía la verdad, pues de hecho, él no disponía de todas las pruebas -que finalmente solo fueron provistas en el marco de la mecánica newtoniana- que permitieran establecer lo bien fundada de dicha elección, que era (en el sentido fuerte del término) una escogencia, un partido que se tomaba de alguna manera, que resultaba de un juicio efectuado a la espera de su validación, por tanto, que estaba por verse:

Estar en la verdad no significa siempre decir verdad. Y es aquí donde la lección del hombre va a aclarar la significación de la obra ${ }^{10}$.

De la misma manera que tener buena salud no es encontrarse en un estado estable cuya perpetuación estaría a prueba de todo riesgo ${ }^{11}$, para un científico

$9 \quad$ El texto de esta conferencia se reproduce en los Estudios de historia y de filosofía de las ciencias, tr. María Luisa Jaramillo, Medellín: Universidad Nacional de Colombia, julio de 1992. pp. 29 ss.

10 Ibíd., p. 38.

11 Canguilhem concluye de todo ello que no es "normal", como uno se lo figura bien a menudo, estar bien de salud. Este tema paradójico lo desarrolló bien al final de la parte complementaria, añadida veinte años después, 
estar en lo verdadero no es encontrarse en un camino que conduce derechito y con toda seguridad a la verdad, lo que exigiría que esta preexistiese a su manifestación, sino que es ser normativo, corriendo el riesgo de escogencias teóricas audaces, que producen rupturas, cuya pertinencia no está de entrada, ni automáticamente garantizada; figurarse que podría ser de otra manera es concebir el devenir del conocimiento como el desarrollo de un sistema completamente racional cuyas condiciones estaban fijas desde el momento de la partida, que siguen un recorrido que conduce necesariamente de verdades en verdades, sin confrontarse con posibles errores y desvíos ${ }^{12}$. En este sentido, a título personal, Galileo estaba bien agarrado por su compromiso propio; era el "sujeto" auténtico de las intervenciones cuyos resultados se consignan en su obra. De manera comparable, en la historia de la biología -dominio al que Canguilhem consagró lo esencial de sus investigaciones- Claude Bernard o Darwin, lejos de estar hundidos en el curso de una lógica inexorable de verdad con respecto a la cual ellos no disponían ya de ningún margen de iniciativa (lo que haría de ellos simples jalones de su desenvolvimiento), han sido verdaderos inventores, creadores de conceptos (el medio interior, para Claude Bernard, la evolución de las especies, para Darwin) cuya pertinencia estaba destinada a ser puesta a prueba, en un ambiente forzosamente polémico de deber-ser. Es por esto que la historia de las ciencias tal y como la practica Canguilhem es,

al estudio sobre lo Normal y lo Patológico, en las consideraciones consagradas a "la enfermedad del hombre normal" (Lo Normal y lo Patológico, Buenos Aires: Siglo XXI, 1975, p. 216).

12 Es lo que lleva a Foucault a afirmar, como conclusión de su texto "La vida: la experiencia y la ciencia": "Este historiador de racionalidades, él mismo tan "racionalista", es un filósofo del error; quiero decir que es a partir del error que él plantea los problemas filosóficos, digamos más exactamente, el problema de la verdad y de la vida" (Michel Foucault. "La vida: la experiencia y la ciencia". Revista de Metafísica y Moral. $90^{\circ}$ año/n. ${ }^{\circ} 1$ Enero-Marzo 1985. pp. 3-14. Traducido por Luis Alfonso Paláu-Castaño. Barcelona, sabático de 1988). Dicho de otro modo, con Canguilhem se aprende a razonar, no de la verdad considerada como una adquisición, sino del error a la verdad, aprehendida dinámicamente como un objetivo por alcanzar, sin garantía de éxito. Esta inversión de perspectiva, o "eversión", resulta del paso de una lógica de la ley, que se aplica a lo que es, a una lógica de la norma, orientada en el sentido de lo que debe o puede ser, con el margen de incertidumbre señalado por la locución "quizás" (peut-être, escrita en francés con el guión): lo posible es a la vez, según la definición que de él da Aristóteles, lo que es y no es, entre presencia y ausencia. "Una saca su sentido, su función y su valor por el hecho de la existencia por fuera de ella de lo que no responde a la exigencia que ella sirve. Lo normal no es un concepto estático o pacífico, sino un concepto dinámica y polémico" (Lo normal y lo patológico, $2^{\text {a }}$ parte, "veinte años después", p. 176). Este pasaje lo comenta Foucault de la siguiente manera: "La norma no se define en absoluto como una ley natural, sino por el papel de exigencia y coerción que es capaz de ejercer con respecto a los ámbitos en que se aplica. La norma, por consiguiente, es portadora de una pretensión de poder. No es simplemente, y ni siquiera, un principio de inteligibilidad; es un elemento a partir del cual puede fundarse y legitimarse cierto ejercicio del poder" (Los anormales, lección del 15 de enero de 1975, México: Fondo de cultura económica, 2000, p. 57). "Una pretensión de poder", "un cierto ejercicio del poder": "poder", correlativo a la intervención de la norma y no a la de la ley, debe entonces entenderse de manera polémica, en oposición a lo que es, en el sentido de la ontología y de la concepción de racionalidad que de acá se deriva (la que define la verdad como "adaequatio rei et intellectus"). Este poder, el que se adosa a la exigencia y a la coerción llevadas por la norma, no consiste en una dominación impuesta a nombre del hecho establecido, sino en el movimiento con miras a un cambio de estado, por tanto de un "puede ser" o de un "quizás". Así como el de norma, el concepto de poder es "dinámico y polémico". 
en el sentido fuerte del término, una historia de científicos que se confrontaron, con los medios de que disponían, y por medio de rectificaciones sucesivas, al debate de lo verdadero y de lo falso, sin saber exactamente hacia dónde iban al momento de partir, salvo que buscaran reinsertar ficticiamente sus procesos en el movimiento retrógrado de lo verdadero.

A primera vista, en Foucault las cosas se presentan de forma completamente diferente; se duda inmediatamente, su reflexión va a pasar claramente por problemas que conciernen "la significación de una obra" como los concernientes a "la lección de un hombre". Cuando intervino en el Instituto de Historia de las Ciencias, con ocasión de las Jornadas de Estudio organizadas en 1969 por el bicentenario del nacimiento de Cuvier por parte de Canguilhem ${ }^{13}$, en el marco de un debate bastante tenso que siguió a su exposición, él generalizó sus apuestas de la siguiente manera:

Pero cuando se trata de estudiar capas discursivas, o campos epistemológicos que comprenden una pluralidad de conceptos y de teorías (pluralidad simultánea o sucesiva) es evidente que la atribución al individuo se vuelve prácticamente imposible. Así mismo, el análisis de estas transformaciones puede difícilmente ser referido a un individuo preciso [...] De suerte que la descripción que trato de hacer debería dejar de lado en el fondo cualquier referencia a una individualidad, o más bien retomar de arriba abajo el problema del autor [...] Pues mi problema es señalar la transformación. Dicho de otra manera, el autor no existe ${ }^{14}$.

13 En la "Introducción", firmada por Canguilhem, a la presentación del conjunto de las intervenciones presentadas en el curso de esas jornadas de estudios, se precisa, con respecto a las discusiones llevadas a cabo en aquel grupo de estudio que personalmente él animaba y en el que habían sido preparadas aquellas jornadas: "Se trata de someter a la prueba de un examen crítico la brillante tesis sostenida por M. Foucault en su obra las Palabras y las Cosas" (Revue dihistoire des sciences, t. XXIII n 1, janvier-mars 1970, Paris, PUF, p. 7). De hecho, en el curso de esas jornadas, la tesis paradójica, iconoclasta, sostenida por Foucault según la cual Darwin está más próximo de Cuvier, a pesar del "fijismo" profesado por este último, que de transformistas como Lamarck, heredero intelectual de Jussieu, y por su intermediario defensor de la teoría de la escala de los seres, teoría que constituía el principal obstáculo para la formación del concepto de evolución de las especies, ha sido discutida por investigadores de los cuales la mayor parte eran cercanos de Canguilhem. Éste por su parte, había ya explicado en "¿Muerte del hombre o agotamiento del cogito?" (aparecido dos años antes) que el punto de vista de Foucault, aunque estaba sujeto a discusión, le parecía perfectamente defendible: "Incluso si se piensa que Foucault no tiene razón en este punto -y personalmente pensamos que él tiene razón- ¿será esta una razón suficiente para acusarlo de haber echado por la borda la Historia?" (op. cit., p. 260). En suma, Canguilhem considera que, en este caso, Foucault está en lo verdadero, incluso si es imposible afirmar con toda certidumbre que lo que dice es verdad, lo que abre un campo de debate. No sin picardía Canguilhem reenvía así a Foucault a su propia normatividad de sujeto de saber. También se podría remarcar que el giro ("éversión") operado en este caso preciso por Foucault, que conduce a interpretar la relación de Cuvier con Darwin, no en un sentido despreciativo, sosteniendo que el fijismo de Cuvier constituyó un obstáculo para la formación de la teoría de la evolución, sino en un sentido positivo, no deja de presentar formalmente una analogía con la rehabilitación del vitalismo efectuada por Canguilhem algunos años antes, en el marco de sus investigaciones sobre el reflejo.

14 Dits et écrits, II n 77, Paris, Gallimard, 1994, p. 60. ‘Traducido por Luis Alfonso Paláu C. para el seminario permanente de Historia de la biología, Escuela de estudios filosóficos y culturales, Facultad de Ciencias Humanas y Económicas, Universidad Nacional de Colombia, Medellín, diciembre 3 de 2002 । Estas afirmaciones, públicamente sostenidas en 1969, recortan y resumen las tesis desarrolladas el mismo año en la conferencia "¿Qué es un autor?" y en la obra la Arqueología del Saber, aparecida igualmente en 1969. 
Continuando su intervención, Foucault llega incluso hasta reprocharse haber utilizado nombres propios como los de Cuvier, Bopp o Ricardo, en Las palabras y las cosas, mientras que el proceso de "transformación" que buscaba evidenciar, sobre cuyo fondo había aparecido la idea de "ciencias humanas", se desenvolvía en un plano en el que no intervienen ni los autores ni las obras. Vista bajo este ángulo, la historia de los conocimientos se presenta como un proceso sin sujeto.

Canguilhem asistía a la discusión que siguió la presentación de esta tesis, discusión en la que, con excepción de una breve anotación consagrada a la temática de la escala de los seres, no intervino ${ }^{15}$. Sería difícil reconstituir el hilo de los pensamientos que han debido sucederse en su espíritu escuchando afirmaciones como las que acabamos de oír que, sin duda por táctica más que por perturbación, se abstuvo de comentar. Pero las personas que siguieron esos debates, y los lectores contemporáneos de sus reseñas que han sido publicadas y conservadas, no han podido y no pueden más que estar afectadas por el contraste entre las posiciones defendidas, por un lado por Foucault, para quien (retomando sus términos) "el autor no existe", y por otra parte, por Canguilhem, atento a la vez, en el conjunto de sus investigaciones en tanto que historiador de las ciencias, a "la significación de la obra" y a la "lección del hombre"; y este contraste produce tanta más perplejidad cuanto que estaba probado que, en puntos precisos de los que se debatían, Canguilhem se ponía de lado de Foucault contra sus objetores y detractores, lo que no podía sino perturbar, por no decir escandalizar a algunas de las personas que trabajaban con él en el Instituto de Historia de las Ciencias; no podían comprender eso que ellas llamaban su "indulgencia" 16 con respecto a tesis que, en el fondo, contravenían el espíritu de sus propias investigaciones, o al menos así les parecía.

Para desenredar este enigma es oportuno volver sobre el diagnóstico hecho por Canguilhem en su artículo "¿Muerte del hombre o agotamiento del cogito?" con respecto al concepto de episteme, diagnóstico favorable en el conjunto, lo que no impide, sin embargo,m que esté abierto a una discusión con respecto a algunos aspectos de esta noción que presentan dificultades u oscuridades. Lo que, en este concepto, le interesa principalmente a Canguilhem es que él intro-

15 Lo esencial de su participación en los debates a los que las jornadas de estudio han dado lugar, estaba luego de la exposición de Francis Courtes, "Georges Cuvier ou lıorigine de la négation"; esa exposición extraordinariamente brillante, sin citar a Foucault, iba en el sentido de su propuesta, en la medida en que, en el polo opuesto de la vulgata que fila a Cuvier en el campo de los tradicionalistas conservadores obstinadamente girados hacia el pasado, él le asignaba a éste un rol creativo en la historia de las ideas sobre la vida y sobre el organismo, por el hecho de haber introducido de manera innovadora el principio de la negatividad, en un sentido cuasi hegeliano. Cuando Foucault tomó la palabra, bien al final de las Jornadas que terminaron con su intervención, Canguilhem, del que todo el mundo sabía que en el fondo estaba de acuerdo con él, permaneció en silencio, sin duda para no hacer pesar en el debate su autoridad personal que nadie habría osado discutir.

16 Recordemos que es el término que emplea Dagognet cuando nos cuenta las reticencias del entorno de Canguilhem con respecto a la acogida favorable que él le concedía a las proposiciones turbadoras de Foucault. 
duce en el análisis histórico lo que llama con un término que regresa en muchas ocasiones en su texto, un principio de "eversión"; por ello se ha de entender el volteo de la mirada que permite desprender el devenir de los conocimientos científicos, a la vez, en el plano de su interpretación, del régimen de las ideas recibidas, por tanto, de la opinión y, en el plano de su desenvolvimiento real, del presupuesto logicista o formalista según el cual la ciencia progresaría en virtud de su propio empuje racional interno, de adquisición en adquisición, sin posibilidad de derivación o de regreso atrás. Pero una vez admitido esto, surge la pregunta:

La episteme, razón de ser de un programa de eversión de la historia, ¿es o no es algo más que un ser de razón? ${ }^{17}$.

Suponiendo que la episteme solo pueda ser un ser de razón, Canguilhem somete esta noción a un examen crítico que no deja de presentar una cierta analogía con el que, en la "Dialéctica trascendental" de la Crítica de la razón pura, Kant le hace sufrir a las "ideas" de la razón, forzosamente ideales y tendencialmente idealistas, el Yo, el Mundo y Dios. Asignar a estas ideas un contenido objetivo cuyo tratamiento quedaría asegurado por una disciplina especial, la metafísica, bajo la forma de un conocimiento sometido a la prueba de la verdad es, según Kant, ir más lejos de lo que lo permiten las capacidades impartidas a la razón $\mathrm{y}$, a término, es legislar en el vacío. Asimismo, ¿no podemos preguntarnos si la noción de episteme no responde objetivamente a ningún contenido asignable, lo que invalida la tentación de hacer de ella el objeto de una ciencia completa bautizada "epistemología"? Pero planteándose esta interrogación, es preciso no olvidar que las ideas de la razón, una vez que se les ha negado un carácter objetivo, no por ello han perdido, desde el propio punto de vista de Kant, toda suerte de legitimidad, eso sí con la condición de que un estatuto diferente les sea atribuido; si no tienen el poder de legislar, esas ideas no por ello dejan de ejercer esa función que Kant llama reguladora que, sin que se reporten directamente a contenidos reales, les hace jugar en el modo del "como si", por tanto a título virtual, a título de posibilidades tomadas en cuenta por sí mismas ${ }^{18}$. En el

17 Op. cit., p. 262.

18 Si la razón no tiene el derecho de afirmar que el Yo, el Mundo y Dios "existen" de conformidad con los datos habituales de la experiencia, nada le impide emplear esas ideas a título puramente indicativo, tratándolas como hipótesis razonables bajo ciertos aspectos. No solamente nada se lo impide sino que ella tiene necesidad de hacerlo pues, en ausencia de esas hipótesis, una síntesis de los conocimientos elaborados por otra parte por el entendimiento en relación con los datos de la sensibilidad, se quedaría indefinidamente diferida. Dicho de otro modo, al ser descartada la tesis según la cual la metafísica es una ciencia exacta, queda abierta la posibilidad de emplearla a título de ficción; yendo más lejos en este sentido, uno podría preguntarse si la función reguladora de las ideas de la razón no está emparentada, en Kant, con el esquematismo de la imaginación ıel Borges que declara que la metafísica es una rama de la literatura fantástica, es pues así un kantiano de estricta observancia, Paláu. Poincaré se inscribe en el surco de esta manera de ver cuando presenta los conceptos científicos como ficciones operatorias, cuya validez es puesta a prueba en su funcionamiento; en tal perspectiva, la epistemología no es nada distinto a una rama de la Poética general. Kant, Poincaré; Valéry, Canguilhem ıy Borgesı se encuentran así colocados en la misma línea. 
mismo sentido, cuando él sugiere que la episteme podría no tener otra realidad que la de un ser de razón, Canguilhem, lejos de considerar que esta noción debe ser descartada, incita a reconsiderar su modo de funcionamiento, es decir, el uso que se puede hacer de ella en el marco impartido a lo que Foucault llama una "arqueología del saber".

Lo que cuestiona Canguilhem es pues precisamente la idea según la cual la episteme podría tener un contenido "objetivo". Luego de haber avanzado la hipótesis de que ella podría ser a lo sumo un ser de razón, él saca la siguiente consecuencia:

Una ciencia es un objeto para la historia de las ciencias, para la filosofía de las ciencias. Paradójicamente, la episteme no es un objeto para la epistemología ${ }^{19}$.

Esto quiere decir que, para la epistemología -es decir, retomando la terminología empleada por Foucault, para la arqueología- ¿ella es otra cosa que un objeto? Ahora bien, si se examina atentamente la manera como procede la tarea arqueológica que tiende a evidenciar, bajo el devenir real de las ciencias, de sus conceptos y de sus problemas, invariantes, se constata que estas son aprehendidas según la modalidad, no de lo real, sino de lo posible. En efecto, ¿con qué tiene que ver directamente la episteme? No directamente con saberes constituidos sino con condiciones de posibilidad de esos saberes, en el sentido en que Kant habla de lo "trascendental" que se mantiene por detrás de todo conocimiento real, aquello de lo que es necesario no precipitarse a concluir que podría él mismo dar su objeto a un conocimiento real: lo trascendental no es otro real que se mantendría más acá de lo real y que cumpliría a su respecto el rol de un fundamento metafísico, sino que representa, incluso en la trama de lo real, lo que constituye en él la parte de lo posible, de lo virtual, de lo tendencial. Es la razón por la que el arqueólogo tiene que vérselas no con cosas sino con discursos sobre las cosas; parte de la base de que las condiciones de posibilidad de un saber no están dadas directamente en lo real al que se reporta ese saber, sino que tiene que ver con la forma discursiva según la cual se construye ese saber, o más bien: puede ser (peut être) construido, es decir: a ser (à être) construido a título de una virtualidad que queda por actualizarse, en el contexto de una actividad o de una práctica de conocimiento efectivo que, al no ser asunto del arqueólogo, interesa específicamente, de manera completamente legítima, al historiador de las ciencias.

Si se ven las cosas bajo este ángulo, los procederes del historiador de las ciencias, que es Canguilhem, y del arqueólogo, que es Foucault, de ninguna manera son alternativos ni excluyentes el uno del otro, porque se mantienen en planos diferentes: el uno tiene que ver con lo real, y más precisamente con lo

19 Op. cit., p. 262. 
real que se está produciendo, bajo la forma de acontecimientos de pensamiento cuya producción está en curso; y el otro se ocupa de posibles que se presentan bajo forma de capas exhibidas, indiferenciadas, indiferentes a la distinción de lo verdadero y de lo falso. Inmediatamente antes de establecer el carácter no normativo de la empresa arqueológica de Foucault, Canguilhem indica:

El concepto de episteme es el de un humus en el cual sólo pueden brotar ciertas formas de organización del discurso, sin que la confrontación con otras formas pueda tener que ver con un juicio de apreciación ${ }^{20}$.

Aquello sobre lo cual solo pueden brotar ciertas formas de organización del discurso, ese "humus" o terreno de formación que es la episteme, no es él mismo un discurso ya organizado siguiendo ciertas formas; si esas formas pueden aparecer sobre él no es a título de gérmenes preformados o de representaciones completamente hechas, sino porque él ofrece la posibilidad, a la espera de que esta se realice, lo que no tiene que ver con su propia determinación. Queda pues por hacer que surjan las formas en cuestión de ese suelo, cultivarlas, siguiendo procesos que tienen que ver no con el arqueólogo, sino con el historiador de las ciencias; este último es conducido así a voltear prioritariamente su mirada hacia las elecciones efectuadas por los científicos, luego de evaluaciones que han orientado sus tomas de partido en un cierto sentido más bien que en otro, y cuyas consecuencias están sometidas a la prueba de la verificación, que diferencia lo verdadero y lo falso; dicho de otro modo: para retomar la terminología empleada por Bachelard, condiciona la repartición entre ciencia caduca y ciencia sancionada, lo que no es la competencia del arqueólogo.

El arqueólogo, que trata de trascendentales y no de las figuras reales emergidas de su puesta en funcionamiento, no se interesa en la distinción de lo verdadero y de lo falso porque, a nivel en que se sitúa su trabajo, esta distinción no tiene un valor probatorio. En una intervención hecha por Foucault, en el marco de las Jornadas de estudio sobre Cuvier, luego de la intervención de Dagognet que había precedido inmediatamente la suya, es presentada esta reflexión a primera vista sorprendente, "eversadora" habría podido decir Canguilhem:

Es necesario distinguir, en el espesor de un discurso científico, lo que es del orden de la afirmación científica verdadera o falsa, y lo que sería del orden de la transformación epistemológica. Que algunas transformaciones epistemológicas pasen por, tomen cuerpo en un conjunto de proposiciones científicamente falsas, me parece ser una constatación histórica perfectamente posible y necesaria ${ }^{21}$.

20 Op. cit., p. 266.

21 Dits et écrits II n ${ }^{\circ} 76$, p. 29. Traducido por Luis Alfonso Paláu C. para el seminario permanente de Historia de la Biología, Escuela de estudios filosóficos y culturales, Facultad de Ciencias Humanas y Económicas de la Universidad Nacional de Colombia, Medellín, noviembre 24 de 2002. 
Dicho de otra manera: que Cuvier haya resultado eventualmente todo falso en el plano de su producción científica propia ${ }^{22}$ no cuestiona para nada el rol de revelador y de índice que le asigna el arqueólogo en el plano, no de la elaboración de conocimientos reales, llamados a ser sometidos a la prueba de lo verdadero y de lo falso, sino del proceso de "transformación" que da a esta elaboración sus condiciones de posibilidad, nada más.

Para comprender mejor el alcance de este razonamiento, no es aberrante servirse de un paradigma retomado de la geografía. En numerosas ocasiones, en particular en la Entrevista aparecida en 1976 en la revista Heródoto ${ }^{23}$, Foucault explicó que se sentía más geógrafo, preocupado por problemas de disposición en el espacio, que historiador, interesado en evoluciones temporales. Por su lado, Canguilhem se había interesado desde muy temprano en las investigaciones en "geografía humana" llevadas a cabo por la escuela de Vidal de La Blache y de de Martonne, en oposición a la tradición germánica de geografía física inspirada por Ratzel y sus sucesores; él había integrado aquellos resultados a su reflexión personal que, rechazando la vulgata ontologizadora, hace pasar a primer plano la consideración axiológica. Este debate entre geógrafos puede aclarar la discusión precedente con respecto a la episteme. Desde el punto de vista de los geógrafos de la escuela alemana, las poblaciones están estrechamente dependientes del suelo que ocupan, y al que están de una vez por todas prendadas o adaptadas $\mathrm{y}$, de alguna manera, clavadas; en esta perspectiva, la geografía explica la historia que no es sino uno de sus avatares. Al introducir el concepto polémico de "geografía humana", los investigadores de la escuela francesa inspirados por Vidal de La Blache denunciaban implícitamente el carácter "inhumano" de esa explicación que somete enteramente las actividades humanas a un determinismo físico al que ellas no pueden sustraerse, y les negaba por consiguiente la capacidad de innovar, de crear, transformando su medio de existencia; ahora bien, este último, si les ofrecía un conjunto de posibilidades, no prejuzga, sin embargo, de las escogencias prácticas indispensables para que esas posibilidades, al menos algunas de ellas, sean efectivamente realizadas o explotadas, lo que tiene en cuenta su iniciativa. Una cosa es entonces el medio ambiente, espacio neutro ocupado por realidades que, estando simplemente allí a título de datos físicos, no están valorizadas en un sentido o en el otro, en la medida

22 Esta sospecha le había dado su hilo conductor a la exposición de Dagognet que, nutriéndola, consideraba quizás que eso constituía precisamente una objeción válida contra lo que sostenía Foucault. Pero este último, en su intervención, le dio vuelta ("everti") completamente a la objeción, haciendo de ella un argumento suplementario en apoyo de su tesis. Canguilhem se debía haber divertido mucho asistiendo a este debate en el que se podía ver una ilustración de la fábula del regador regado. Que Cuvier haya podido eventualmente equivocarse no afecta para nada el rol que se le reconoce por parte de la arqueología.

23 "Questions à Michel Foucault sur la géographie”, Dits et écrits III n. ${ }^{\circ}$ 169, Paris, Gallimard, 1994, p. 28 ss. M. Foucault. Microfísica del poder. Madrid: la Piqueta, 1979. pp. 111 ss. Ver también "Des questions de Michel Foucault à Hérodote", ibid. n 178, p. 94- 95. 'se traducen aquí como anexo 2, Paláus 
en que ellas no ofrecen perspectivas de escogencias completamente trazadas; otro es el medio ocupado y explotado en la práctica por poblaciones que, en particular gracias a los medios artificiales provistos por la técnica, disponen ese espacio para fines de utilidad, fines que de entrada no estaban inscritos en la naturaleza de las cosas, sino que tenían que ver con su responsabilidad de introducirlos allíi ${ }^{24}$. Este análisis ampliado, más allá de lo humano y de las formas propiamente técnicas que toma su actividad, al viviente considerado en general, recorta la distinción hecha, en etología, por Uexküll entre Umgebung y Umwelt: el primero constituye un entorno natural de hecho, objetivo, como tal indiferente a las prácticas efectivas que allí se desenvuelven bajo la responsabilidad de los sujetos que las ejecutan, mientras que el segundo es un mundo de signos y de significaciones, informado por las necesidades y las tendencias de sus habitantes que se lo han adaptado con miras a llevar allí su vida propia ${ }^{25}$, y no tanto que ellos se hayan adaptado a él.

Cuando Foucault introduce la noción de arqueología, que hace referencia a un basamento que está más profundo que lo edificado encima de él, apunta su atención al entorno global que da un lugar de acogida a actividades de conocimiento que pueden aparecer dentro de ese marco, sin que su aparición obedezca a un principio de determinación sometido a la ley objetiva de las cosas. Dicho de otro modo: lo que él llama la episteme de una época no es el conjunto de los conocimientos, ya preformados, que podrán ser elaborados en esa época; ella representa únicamente su trascendental, ese "ser de razón" que constituye a fin de cuentas su condición de posibilidad ${ }^{26}$. El arqueólogo se remonta hasta ese marco, humus en el que queda por hacer que se presenten realmente esas formaciones cognitivas que son las ciencias propiamente dichas, con respecto a las cuales, interrogantes como los de "la significación de la obra" o el de "la lección del hombre" merecen ser formulados.

Se comprende, entonces que, si bien la arqueología ha puesto entre paréntesis la consideración de la normativa y de la subjetividad, la historia de las ciencias

24 Lucien Febvre, en su obra sobre La terre et liévolution de lihumanité (Introduction géographique à lihistoire), aparecida en 1922 en las ediciones Albin Michel en la colección Liévolution de lihumanité, ‘La tierra y la evolución humana. Introducción geográfica a la historia. México: UTEHA, 1955) se sirvió de la expresión "posibilismo", como alternativa a "determinismo", para designar la orientación nueva comunicada por Vidal de Lablache a las investigaciones de los geógrafos.

25 Como la hembra de la garrapata fecundada que, estando colgada en una rama de árbol, sólo es sensible en lo que la rodea a un cierto olor a mantequilla rancia que le señala el paso, al pie del árbol, de un mamífero de sangre caliente sobre el que se deja caer para penetrar su epidermis y poner allí sus huevos, luego de lo cual, su destino de garrapata se ha realizado, y sólo le queda morir; el mundo exterior para ella se reduce a ese olor; considerado en su globalidad sólo es, en la perspectiva que es la suya, un "ser de razón" privado de objetividad, y como tal irrepresentable.

26 ıbrillantísima explicación de algo que pocos han podido entender: que exista un a priori histórico.... Cfr. la Arqueología... Paláus. 
pueda, sobre las bases así exhumadas, reintroducir esta consideración con miras a mostrar cómo, del humus primordial, han efectivamente salido tales o cuales conocimientos reales, a lo largo de procesos que no estaban enteramente prefigurados en sus condiciones de partida, condiciones que es necesario no ir a asimilarlas con ningún tipo de fundamento, ya sea lógico o arqueológico. Desde entonces, el trabajo de Foucault y el de Canguilhem ya no son contradictorios, incluso aparecen como complementarios el uno del otro.

Esta complementariedad se ha hecho posible por el hecho de que la arqueología no saca a luz para nada a un sistema primero de conocimientos, conocimientos de esos de antes del conocimiento, a partir de los cuales los científicos no tendrán luego más que efectuar gradualmente el desarrollo. Si un tal sistema existiese, de entrada estaría estructurado y ordenado siguiendo normas destinadas a ser aplicadas de manera conforme o no conforme. Ahora bien, la episteme, tal como la trata el enfoque de Foucault, se presenta de manera completamente diferente: neutra axiológicamente, en el sentido en que no está sometida a criterios permanentes de comprobación de su valor propio de verdad; ella solamente ofrece un campo a la investigación de verdades que no están prefiguradas en ese campo. La episteme hace posible la prueba de la verdad, pero ella no está sometida a esa prueba que, por consiguiente, presenta un carácter, no absoluto, sino relativo; en efecto, solo existe verdad en relación con un contexto epistémico dado, contexto con respecto al cual hay o puede haber verdad, sin que haya lugar para interrogar a dicho contexto sobre su valor propio de verdad.

En "¿Muerte del hombre o agotamiento del cogito?”, Canguilhem plantea, de paso, la cuestión de saber si ese relativismo -pero quizá sería preferible hablar de un relacionismo, que desemboque en una puesta en red de la cuestión de la verdad que permita recontextualizarla- no tendrá que ver con el que da su inspiración al proceder culturalista bajo la forma que le han dado los sociólogos y antropólogos norteamericanos. Esta andadura conduce a la revelación de invariantes, como, por ejemplo, la "personalidad de base", revelación que permite medir el grado de integración de los individuos a la totalidad social de la que ella constituye el paradigma identificatorio. Asimismo, si se toma en serio el acercamiento, parecería que el trabajo del conocimiento, visto desde el punto de vista del arqueólogo, se desenvolvería sobre el fondo de una "episteme de base" que constituiría, según los términos empleados por Canguilhem, "su sistema universal de referencia en tal época, cuya diferencia es la única relación que mantendría con el que le sigue"27; este universal de referencia, al solo ser universal para su época, se le supone pues que tiene valor en sí, independientemente de una posibilidad de evaluación que lo haga salir de su límites propios. Pero la

27 "Mort de lihomme ou épuisement du cogito?", p. 266. 
hipótesis de la que se reclama este enfoque, tan pronto se lo plantea, debe ser descartada. En efecto, hay una diferencia esencial entre la episteme y lo que el culturalismo estadounidense coloca bajo la categoría de personalidad de base. Bajo esta categoría se encuentra un paradigma que, sin haber sido él mismo normado a partir de los criterios de evaluación que exceden su naturaleza propia, norma los comportamiento particulares de los individuos a los que se reporta en un marco cultural dado; neutro axiológicamente si se lo considera en sí mismo, también es en el plano de su uso, separador, generador de conformismo, por tanto no neutro, que es a lo que lo destina su naturaleza misma de paradigma; según los términos empleados por Canguilhem, él representa "el concepto a la vez de un dato y de una norma que una totalidad social impone a sus partes para juzgarlas, para definir la normalidad y la desviación"28. Ahora bien, la episteme, que ofrece un campo, es decir, un conjunto de disponibilidades indispensables para las operaciones efectivas del conocimiento, no legisla sobre el uso de esas disponibilidades y, por consiguiente, no le provee a las operaciones que hace posibles (a las que les ofrece sus condiciones necesarias pero no sus condiciones suficientes) modelos estándares que permitan discriminar sus resultados; la neutralidad axiológica propia de este campo se extiende al conjunto de las operaciones a las que da lugar, es decir, que él no le impone nada a estas normas de verdad preconstituidas, independientes de su puesta en funcionamiento efectivo. Sobre el humus de ese campo brota, no de lo normal apreciado como tal con relación a patrones dados ne varietur, sino de lo normativo, es decir un trabajo prospectivo de invención cuya responsabilidad la tienen personalmente los científicos, lo que los conduce en esta ocasión a "estar en lo verdadero" o, más bien, a meterse en ello, por su propia cuenta y riesgo, incluso sin saber y sin poder saber, propiamente hablando, lo verdadero por adelantado. Por acá mismo se reanuda una relación entre episteme y posición de sujeto; si no existe sujeto fundador que se mantendría tras la episteme -y en este sentido es claro que ella no tiene sujeto- hay lugares delante de ella para la acción efectiva de sujetos productores de conceptos y trabajadores de la prueba, acción a la que ella ofrece, no modelos prefabricados sino disponibilidades. El sujeto no ha sido suprimido, sino que cambió de lugar y, por lo mismo, de naturaleza ${ }^{29}$.

\footnotetext{
28 Ibídem.

29 Es lo que conduce a Foucault, bien al final de su texto "la Vida: la Experiencia, la Ciencia" a preguntarse: "¿No será que toda la teoría del sujeto debe ser reformulada desde que el conocimiento, más bien que abrirse a la verdad del mundo, se enraíza en los "errores" de la vida?" (Dits et Ecrits, t. IV, Paris, Gallimard, 1994, p. 776 (tr. Paláu, Sociología 18, Medellín: Universidad Autónoma Latinoamericana, Julio/1995. pp. 15-16.ı). Desde entonces, la partición entre "una filosofía de la experiencia, del sentido, del sujeto" y "una filosofía del saber, de la racionalidad y del concepto" mencionada por Foucault en la introducción de su texto (ibid., $1^{\text {a }}$ col. p. 8) deja de tener un valor explicativo; una concepción del conocimiento y de su historia como la de Canguilhem, que hace pasar al primer plano la vida de los conceptos, lejos de evacuar definitivamente la referencia al sujeto, conduce a repensar el estatuto de esa referencia, por tanto a asignarle una nueva posición al sujeto.
} 
Se puede pues afirmar que, incluso durante el período en que había alejado de sus preocupaciones la consideración de un sujeto de las normas, no solamente sujetado a normas, sino creador de normas, y como tal inclinado a lo que llamará más tarde "el cuidado de sí", Foucault no invalidó de ninguna manera la cuestión del sujeto, sino que preparó una manera novísima de abordarlo, que pondrá en funcionamiento durante el período ulterior en que esa cuestión del sujeto regresará, tomada en sí misma, al primer plano de su atención. Para caracterizar ese nuevo abordaje, se puede retomar la fórmula de la que se servía Canguilhem en el título de su artículo, "Agotamiento del cogito", que no significa, como lectores demasiado apresurados se lo han figurado, la desaparición del sujeto, ni siquiera -tomando esta fórmula al pie de la letra- la "muerte del hombre". Cuando Foucault explica que, en el plano propio de la arqueología, el hombre solo ocupa, a título de objeto de conocimiento, la posición de una formación derivada, destinada, una vez llegue el momento, a borrarse como una figura trazada en la arena a la que la marea viene a recubrir, se dedica al género de especulaciones que Althusser, sirviéndose de referencias y de medios diferentes, ponía bajo la categoría de "humanismo teórico"30; pero, poniendo en su sitio este tipo de especulaciones que, dice él, cumplieron su ciclo, él libera el campo donde podrá aparecer una noción de un tipo completamente diferente, cuya caracterización toma prestada de Nietzsche, bajo la denominación "superhombre". Ahora bien ¿qué es el superhombre? Es el tipo de sujeto que debe salir del "agotamiento del cogito", por tanto, un sujeto que ha dejado de ser sustancial, res cogitans, y que ya no juega el papel de un fundamento teórico, sino que se ha vuelto sujeto práctico, sujeto de sus actividades, en el primer rango de las cuales está la de hacerse a sí mismo, o hacer de sí su obra propia, a prueba de los valores de los que ha hecho elección, por su propia cuenta y riesgo, no completamente solo sino con los otros, y eventualmente en conflicto con ellos.

Canguilhem también era un lector de Nietzsche, y su concepción de la normativa del sujeto converge (por otras vías distintas a las seguidas por Foucault) en la de un sujeto práctico, sometido a la exigencia del deber ser bien simplemente porque él ya no es completamente a la manera de una cosa, sino que él tiene que ser, debe hacerse, por su propia actividad creadora, que en nada está sometida a un determinismo natural, incluso si ese determinismo le ofrece el fondo sobre el cual le queda trazar su camino propio bajo su responsabilidad. En momentos en que preparaba su tesis de Medicina, que después constituyó el cuerpo principal de la obra sobre Lo normal y lo patológico, Canguilhem se había

30 Por "humanismo teórico", hay que entender la especulación ordenada a la representación de una "esencia humana" cuya realidad estaría dada en lo absoluto, a título de una "naturaleza" completa, tratada, para retomar los términos empleados por Spinoza, "tanquam imperium in imperio". Es de esta representación, aún dominante en Feuerbach, que Marx ha debido desprenderse para elaborar su materialismo histórico. 
interesado en filósofos neokantianos de la escuela de Heidelberg ${ }^{31}$; en su espíritu, su manera singular de razonar había recortado las enseñanzas que había sacado de Renouvier y de Hamelin, y que iban en el sentido de lo que se puede llamar un "posibilismo" 32 , poniendo de presente un punto de vista axiológico, como alternativa a un "determinismo", que tenía mucho más que ver con un punto de vista ontológico, es decir, con una "filosofía de cosas"33. Que el hombre, como por lo demás el conjunto de los seres vivos, evoluciona en un mundo lleno de cosas que pueden indiferentemente ${ }^{34}$ serle útiles o dañinas, es algo que no se puede negar; pero esto no autoriza a llevarlo al rango de una cosa al lado de las otras, así solo sea porque él, a su manera, tiene a bien ser la cosa que él es; esta manera se distingue por su capacidad de cambiar su medio de existencia transformándolo por medio de la técnica y, cuando se requiere, cambiando de medio, una capacidad de la que los seres vivos no disponen, al menos no en el mismo grado. Canguilhem, generalmente avaro en consideraciones de orden general, ha priorizado en particular esta consideración -a la que le daba la forma de una "exigencia" (palabra que regresa a menudo cuando concentra las apuestas de su orientación filosófica personal)- en la conclusión de su conferencia sobre "el Cerebro y el pensamiento", donde introduce, de manera bastante inesperada la referencia a Spinoza, un filósofo que, en razón del sustancialismo y del necesitarismo que se le acredita frecuentemente, no estaríamos inclinados a colocar bajo la categoría de "posibilismo"35. Es la razón por la que, cuando se refiere a

31 Windelband \& Rickert, principales representantes de esa escuela, efectúan una relectura original del kantismo, que lo reduce en el fondo a una filosofía del juicio y de los valores, punto de vista en el cual la consideración del "deber-ser" (Sollen) tiene prioridad sobre la del ser (Sein). Por ejemplo, una representación verdadera es la que debe ser pensada, una acción buena es la que debe ser realizada, una cosa bella es la que debe agradar, etc., en el sentido en que "debe ser" no remite a una obligación, o al juego de un automatismo, sino que representa un posible que, una vez valorizado, falta por hacerlo pasar a los hechos; esta puesta en funcionamiento tiene que ver, según estos pensadores, no con la responsabilidad de sujetos personales sino de la cultura histórica de una época y de un lugar dados considerada, no como un sistema obligatorio, sino como un conjunto que está buscando hacerse su lugar, ordenarse, orientarse siguiendo sus tendencias propias. En la tesis de Medicina de 1943, Canguilhem cita de paso una fórmula del filósofo austríaco Reininger, extraída de su obra Wertphilosophie und Ethik (1939), que resume esta orientación de pensamiento: "Unser Weltbild ist immer zugleich ein Wertbild" ("Nuestra visión del mundo es siempre al mismo tiempo una imagen de valor") (Le Normal et le pathologique, Paris, PUF/Quadrige, 1988, p. 117). Canguilhem adoraba este género de fórmulas lapidarias.

32 Recordemos que esta expresión es la que utiliza Lucien Febvre para caracterizar la orientación propia de las investigaciones en "geografía humana".

33 Renouvier se servía de la expresión "filosofía de cosas" para caracterizar la manera de pensar propia de Spinoza.

34 Indiferentemente, en la perspectiva propia de ese mundo de cosas, si es que se le puede imputar a este último ral mundo una perspectiva; es al sujeto, humano o simplemente viviente al que le corresponde hacer la diferencia entre lo que, viniendo de este mundo, le es útil y lo que le es dañino, corriendo por supuesto el riesgo de equivocarse.

35 El lector de la Ética que se detiene en la primera parte de la obra tropieza, en el Apéndice de ella donde la idea de finalidad natural es definitivamente desacreditada desde un punto de vista racional, con la fórmula "omnia sunt praedeterminata", "todas las cosas están predeterminadas" en el sentido en que obedecen al 
Spinoza para concluir su exposición sobre "el Cerebro y el pensamiento", no es desde el ángulo teórico que pudiera ser puesto bajo la rúbrica "la significación de la obra" que Canguilhem lo hace; "Spinoza" -y cuando pronuncia el nombre de Spinoza Canguilhem piensa, y piensa muy fuerte, en Cavaillès que se había declarado en muchas ocasiones "spinozista"- es ante todo para él "la lección del hombre", un hombre que ha sido un héroe del pensamiento no solamente en el plano de sus elecciones teóricas, sino en el de la vida práctica, y propiamente de la política, de sus violencias y de sus azares.

Entonces, "Spinoza", antes que un nombre pudiendo servir de etiqueta a una doctrina que tiene su lugar en la historia de los sistemas de pensamiento, es el representante de una actitud o, para retomar una palabra que le encanta a Canguilhem, una manera de obrar filosófica, una cierta forma de orientarse, de perfilar sus intervenciones, de ser "normativo", no solamente en el pensamiento sino también en la vida:

En cuanto a la filosofía, su tarea propia no es la de aumentar el rendimiento del pensamiento sino el recordarle el sentido de su poder. Asignarle a la filosofía la tarea específica de defender al Yo como reivindicación intransferible de presencia-vigilancia es reconocerle solamente el papel de la crítica. Por lo demás esta tarea de negación no es de ninguna manera negativa, pues la defensa de una reserva es la preservación de las condiciones de posibilidad de la salida (...) Defender su circunspección impone salir de ella cuando se requiera, como lo hizo Spinoza. Salir de su reserva es hacerlo con su cerebro, con el regulador viviente de las intervenciones que actúan en el mundo

principio de causalidad cuya fuente última se encuentra en Dios, es decir en la naturaleza; si es curioso, puede entonces preguntarse si, una vez formulada tal tesis, el proyecto mismo de una "ética" -es decir de una teoría de la acción- no se encuentra por ello mismo invalidado, lo que por lo menos sería extraño en el comienzo de una obra titulada precisamente Ética, y no Lógica o Metafísica. Su sorpresa comienza a disiparse cuando, avanzando en su lectura descubre que, a comienzos de la cuarta parte de la obra, Spinoza reintroduce (con la noción de lo que es útil en el doble sentido de lo útil propio y de lo útil común) la consideración de la finalidad, es verdad que bajo un novísimo aspecto; se trata en efecto entonces de una finalidad que no está inscrita en la naturaleza de las cosas, sino que tiene que ver con la actividad humana, en tanto que esta, llevada por el impulso del conatus, que empuja a cada ser a perseverar en su ser -empresa cuyo éxito no está garantizado a priori- está sometido a la vez a la ley innovadora y mortífera de la afectividad y del deseo, a los juegos de Eros y de Thanatos estaríamos tentados a decirlo en el lenguaje de Freud. Aparece entonces que vivir -y escribir una "Ética" es precisamente proponer un arte de vivir- no es un estado, una propiedad de cosa, sino una actividad, como tal polarizada, polémica, es decir confrontada permanentemente con la alternativa entre preferir y excluir, de donde resulta la separación de lo normal y de lo patológico. La referencia a "valores", incluso si ella opera con frecuencia sobre el fondo del conocimiento de primer género, es decir de la imaginación (lo que impide atribuirle un carácter racional) reencuentra entonces un interés filosófico; y de hecho, las dos últimas partes de la obra de Spinoza están consagradas al esfuerzo con miras a volver a trabajar la creencia en valores, salida espontáneamente de los procedimientos de la imaginación que conducen a figurarse que se desean ciertas cosas porque se juzga que son buenas, mientras que a la inversa uno juzga que son buenas porque se las desea, con el fin de elevar la convicción en el valor de los valores a nivel de la razón, lo que equivale a fin de cuentas a reconciliar afectividad y racionalidad, última palabra de la sabiduría. Resumamos: si las consideraciones sobre las sustancias, los atributos y los modos que Spinoza ha escogido como punto de partida de su demostración, parecen obstaculizar o convertirse en una pantalla al desarrollo de una axiología, filosofía de los valores o del juicio, en realidad ellas son jalones preparatorios para la elaboración de ésta, puesto que no se ve cómo una reflexión "ética", por tanto girada hacia la práctica, podría eximirse de ella. 
y en la sociedad. Salir de su reserva es oponerse a toda intervención extraña sobre el cerebro, intervención que tiende a privar al pensamiento de su poder de reserva en última instancia ${ }^{36}$.

Bajo una forma concentrada -una extrema concentración es la marca distintiva del "estilo" de Canguilhem ${ }^{37}$ - esta declaración vehicula toda suerte de reservas. Cuando Canguilhem le asigna a la filosofía, bajo la responsabilidad de un "Yo" de vigilancia ${ }^{38}$, una "tarea de negación que no es de ninguna manera negativa", explota una concepción original de la negación que remite a la vez al Ensayo con miras a introducir en filosofía el concepto de negativo de Kant, a la interpretación no-hegeliana de la dialéctica como "heterología" de Ricket, y a la "filosofía del no" de Bachelard: pensar, como todas las otras actividades vitales, es confrontarse con valores negativos, superar obstáculos; por tanto, en el sentido fuerte del término: "resistir", esta última palabra, remitiendo a una gama de comportamientos que van desde mantener la circunspección en la adversidad, a la de oponerse, incluso por la fuerza, si es necesario. Cuando evoca la necesidad en la que el filósofo se encuentra, en este caso, de "salir de su reserva", Canguilhem piensa evidentemente en el compromiso de Cavaillès en el movimiento de la resistencia contra el régimen de Vichy y la ocupación alemana, un compromiso que le costó la vida, y en su propio compromiso con ese movimiento en el que había seguido su ejemplo, como no ha cesado de recordarlo, un ejemplo cuyo alcance es simultáneamente político y filosófico, no siendo posible el uno sin el otro. Por discreción, por "reserva" -la reserva era un carácter distintivo de la personalidad de Canguilhem- la conclusión de la conferencia sobre "el Cerebro y el pensamiento" no hace expresa referencia a este compromiso que Canguilhem mismo practicó, como filósofo, arriesgando el pellejo en los montes de Auvergne, pero lo evoca sirviéndose de una parábola tomada de una de las biografías de Spinoza compuestas luego de su muerte. Esa parábola, de la que no se sabe si se refiere o no a hechos reales o legendarios -por lo demás en su época, Spinoza era alguien a propósito de quien se podía efectivamente contar este género de historias, ya fueran verdaderas o falsas-, concierne la expedición solitaria que habría llevado a cabo Spinoza por las calles de La Haya, blandiendo una pancarta en la que estaban escritas las palabras: "Ultimi Barbarorum"; el acontecimiento habría ocurrido en 1672, en el momento del asesinato de los hermanos Witt, los Grandes Pensionistas del régimen republicano instaurado algunos años antes, en el que parece-quizá

36 "Le cerveau et la pensée" (1980), reproducido como introducción al volumen colectivo Georges Canguilhem philosophe historien des sciences, Paris, Albin Michel, 1993, p. 31. G. Canguilhem. "El cerebro y el pensamiento", publicado en la revista Sociología 17 de la Universidad Autónoma Latinoamericana, Medellín, Junio de 1994, p. 23, $2^{\text {a }}$ \& $3^{\mathrm{a}} \mathrm{col}$. ıcolgado en la red por la Revista colombiana de psicología, $n^{\circ}$ 5-6, 1996. Bogotá: Universidad Nacional de Colombia).

37 ıestilo que con razón, a un poeta de los nuestros, le parecía "pedregoso", Paláus.

38 "El Yo no está con el mundo en relación de sobrevuelo sino en relación de vigilancia" (ibíd., p. 22, 3. ${ }^{a}$ col.). 
sea otra leyenda- había participado a título de Consejero; y una vez descubierto el asunto, había hecho su apología en su Tractatus Teológico-político publicado anónimamente en 1670, una llamarada que se difundió inmediatamente por toda Europa. Este proceder, a la vez temerario y simbólico, presenta una significación filosófica en la medida en que remite a la existencia de un "Yo" que no se contenta con adoptar, con respecto a la realidad, una posición de sobrevuelo, sino que, incluso en sus redes, ejerce una función de "vigilancia". Pero en el mismo movimiento aclara el conjunto de la filosofía de Spinoza cuyo alcance es revelado plenamente:

En suma, esta filosofía que refuta y rehúsa los fundamentos de la filosofía cartesiana, el cogito, la libertad en Dios y en el hombre, esta filosofía sin sujeto, muchas veces asimilada a un sistema materialista, esta filosofía vivida por el filósofo que la pensó, imprimió a su autor el empuje necesario para insurgirse contra el hecho cumplido. La filosofía debe dar cuenta de tal poder de empuje ${ }^{39}$.

Visto bajo este ángulo, Spinoza es por excelencia un filósofo "normativo" que, profesando "el agotamiento del cogito", abre un campo de intervención a un nuevo "Yo", definido 'por su "poder de empuje"40 que lo conduce a "insurgirse contra el hecho cumplido".

Este desvío por Spinoza, un pensador en el que parece que Foucault nunca se interesó, conduce a él por un sesgo inesperado. En efecto, ¿qué es Spinoza para Canguilhem? Es el filósofo que detenta y ejerce un "poder de empuje" que lo impulsa a insurgirse, a resistir. Pues resulta que, en sus últimas producciones teóricas, y en las intervenciones que hacía en caliente sobre las cuestiones de actualidad, Foucault le dio una particular importancia a la noción de "resistencia". Incluso se puede avanzar que fue esta noción la que hizo la transición entre su reflexión sobre la cuestión del poder y la del sujeto, que pasó a primer plano en sus últimos trabajos. La idea que desarrolla Foucault a este respecto es que la resistencia no es un fenómeno aislado, y como tal accidental, porque su necesidad es consustancial al ejercicio del poder, del que constituye la otra cara; es la razón por la que no hay forma concebible de poder que no comporte en su estructura la tendencia a resistirle; en este sentido, al nivel que juegue, el poder, lejos de constituir un sistema cerrado, opaco y sin fallas, deja siempre lugar a la tendencia a enfrentarlo, y en el límite a derrocarlo. Ahora bien, este lugar es precisamente aquel por el que se insinúa el sujeto salido del agotamiento del cogito; un sujeto que no reivindica una identidad sustancial independiente, por tanto, absoluta, sino que, ocupando los intersticios de la realidad, desapretando las "mallas del poder", se hace por ello mismo normativo, es decir: ya no detentador de una subjetividad de la que dispondría como si se tratase de un

$39 \quad$ Ibíd., p. 23, 2. ${ }^{\mathrm{a}} \mathrm{col}$.

40 Esta expresión "poder de empuje" restituye lo más cerca posible la significación de la noción de conatus. 
hecho cumplido, sino autor responsable de sí mismo, creador de una subjetividad que se ha vuelto su propia obra. De esta orientación en dirección a los problemas de la normativa y de la subjetividad, que se ha vuelto dominante en los últimos trabajos de Foucault, Canguilhem lo había pronosticado en 1967, lo anunció en la conclusión de su artículo "¿Muerte del hombre o agotamiento del cogito?". Luego de haber subrayado el interés de las nuevas luces aportadas por Las palabras y las cosas sobre la formación de la idea de "ciencias humanas", propone en las últimas líneas de su texto la siguiente hipótesis:

A menos de que, no tratándose ya aquí de la naturaleza y de las cosas, sino de esa aventura creadora de sus propias normas a la que el concepto empírico-metafísico de hombre, sino la palabra misma, pudiera cesar un día de convenir, no había diferencia que hacer entre el llamado a la vigilancia filosófica y la puesta al día -un día crudo mucho más que cruel- de esas condiciones prácticas de posibilidad ${ }^{41}$.

Se requería mucha lucidez y vigilancia filosófica para desentrañar de manera premonitoria que, en la reserva de los análisis desarrollados por Foucault en Las palabras y las cosas donde la problemática del saber parecía ocupar una posición preeminente, se encontraban las preocupaciones propias de una "filosofía práctica", preocupaciones filadas sobre actividades en las que deben participar "sujetos", sujetos de vigilancia y no de sobrevuelo. En la continuación de su obra, Foucault dará cada vez más importancia efectivamente a tales consideraciones, lo que Canguilhem había presentido de entrada. 41 Canguilhem, G. “¿Muerte del hombre o agotamiento del cogito?” In: Análisis de Michel Foucault. BsAs: Editorial
Tiempo Contemporáneo, 1970. 